\title{
Metamaterial characterization using Boltzmann's kinetic equation for electrons
}

\author{
Novitsky, Andrey; Zhukovsky, Sergei; Novitsky, D.; Lavrinenko, Andrei
}

Published in:

Proceedings of Metamaterials 2013

Link to article, DOI:

10.1109/MetaMaterials.2013.6809066

Publication date:

2013

Document Version

Peer reviewed version

Link back to DTU Orbit

Citation (APA):

Novitsky, A., Zhukovsky, S., Novitsky, D., \& Lavrinenko, A. (2013). Metamaterial characterization using Boltzmann's kinetic equation for electrons. In Proceedings of Metamaterials 2013 (pp. 400-402). IEEE. https://doi.org/10.1109/MetaMaterials.2013.6809066

\section{General rights}

Copyright and moral rights for the publications made accessible in the public portal are retained by the authors and/or other copyright owners and it is a condition of accessing publications that users recognise and abide by the legal requirements associated with these rights.

- Users may download and print one copy of any publication from the public portal for the purpose of private study or research.

- You may not further distribute the material or use it for any profit-making activity or commercial gain

- You may freely distribute the URL identifying the publication in the public portal

If you believe that this document breaches copyright please contact us providing details, and we will remove access to the work immediately and investigate your claim. 


\title{
Metamaterial Characterization Using Boltzmann's Kinetic Equation for Electrons
}

\author{
A. Novitsky ${ }^{1}$, S. V. Zhukovsky ${ }^{2}$, D. Novitsky ${ }^{3}$, and A.V. Lavrinenko ${ }^{2}$ \\ ${ }^{1}$ Belarusian State University, Nezavisimosti Ave. 4, 220030, Minsk, Belarus \\ ${ }^{2}$ DTU Fotonik, Technical University of Denmark, Ørsteds Plads 343, DK-2800, Kgs. Lyngby, Denmark \\ ${ }^{3}$ B.I. Stepanov Institute of Physics, Nezavisimosti Ave. 68, 220072, Minsk, Belarus \\ novitsky@bsu.by, sezh@fotonik.dtu.dk
}

\begin{abstract}
Statistical properties of electrons in metals are taken into consideration to describe the microscopic motion of electrons. Assuming degenerate electron gas in metal, we introduce the Boltzmann kinetic equation to supplement Maxwell's equations. The solution of these equations clearly shows the resonant behavior of electronic response to an external electromagnetic field. We demonstrate the approach for planar and circular geometries of the metamolecules.
\end{abstract}

\section{INTRODUCTION}

Interaction of electromagnetic radiation with a metamaterial (MM) is the cornerstone theoretical problem addressed in numerous works [1-6]. On the other hand, it is well known that the properties of bulk dielectrics and metals can be derived using a simple Drude-Lorentz microscopic model. Generalizing this model to describe the behavior of electrons in a more complex geometry such as an elementary cell of a MM is thus promising because it lets the effective properties of MMs be recovered ab initio. This approach was previously applied to planar chiral MMs where the elementary cell comprises two asymmetric ring segments [7]. It was shown that the resonance in the MM response appears due to two mutually opposing forces: (i) the Coulomb force from the charges at the tips of the ring segments; (ii) the electromotive force owing to the excited currents flowing along these segments.

In this paper, we put forth a systematic microscopic approach applicable to a much wider class of MMs including metallic inclusions with arbitrary shapes. The approach is based on the kinetic Boltzmann equation for electrons. The general equations for the effective properties of MMs are derived and applied to two simple but widespread inclusion shapes, namely, a metallic bar and a metallic ring segment.

\section{Kinetic Equation For ELECTROnS}

Let us consider metallic inclusions (one or more per MM unit cell) inside a dielectric matrix. In an external electromagnetic field the electrons in metal start to acquire inhomogeneous distribution. The non-equilibrium state of the electron gas satisfies the kinetic Boltzmann equation for the electron density $f(\mathbf{p}, \mathbf{r}, t)[8]$ :

$$
\frac{\partial f}{\partial t}+\mathbf{v} \cdot \nabla f+\mathbf{F} \cdot \frac{\partial f}{\partial \mathbf{p}}=\operatorname{St}(f)
$$

where $\mathbf{p}$ and $\mathbf{v}$ are the electron momentum and velocity, respectively; $\mathbf{F}=e \mathbf{E}+(e / c) \mathbf{v} \times \mathbf{B}$ is the Lorentz force affecting electrons with charge $e ; \operatorname{St}(f)$ is the collision integral taking into account the probability of electronelectron, electron-phonon, and electron-impurity collisions. The term with $\nabla f$ can be omitted since it represents spatial dispersion for electrons, which is negligible for MMs unless the inclusion sizes are sub-nanometer [9].

Let us suppose that the full density function $f$ can be written as the sum of the equilibrium density function $f_{0}(\mathbf{p})=\left(\exp \left[\left(p^{2} / 2 m-\mu\right) / k_{B} T\right]+1\right)^{-1}$ (the ordinary Fermi-Dirac distribution of the degenerate electron gas) and a non-equilibrium term $\delta f(\mathbf{p}, \mathbf{r}, t)$. Here $m$ is the electron mass, $k_{B}$ is the Boltzmann constant, and $T$ is the temperature. Electron collisions can then be effectively introduced using the decay frequency $\gamma$ in metals, so $\operatorname{St}(f)=-\gamma \delta f$. Thus, Eq. (1) reduces to an equation for $\delta f$ in the form

$$
\frac{\partial \delta f}{\partial t}+e\left(\mathbf{E}_{\mathrm{inc}}+\langle\mathbf{E}\rangle\right) \cdot \frac{\partial f_{0}}{\partial \mathbf{p}}=-\gamma \delta f
$$


where $\mathbf{E}_{\mathrm{inc}}$ is the electric field of the incident wave and $\langle\mathbf{E}\rangle$ is the electric field arising due to the induced charges and currents in the whole MM. The term with $\mathbf{B}$ disappears because $[\mathbf{v} \times \mathbf{B}] \cdot\left(\partial f_{0} / \partial \mathbf{p}\right) \sim[\mathbf{p} \times \mathbf{B}] \cdot \mathbf{p}=0$.

The electric field $\langle\mathbf{E}\rangle$ satisfies Maxwell's equations with the electric charge density $\rho(\mathbf{r}, t)$ and electric current density $\mathbf{j}(\mathbf{r}, t)$ as sources. The solution of the Maxwell equations is the volume integral over the metal volume

$$
\langle\mathbf{E}\rangle=-\int_{\text {metal }} G\left(\mathbf{r}-\mathbf{r}^{\prime}\right)\left(\nabla^{\prime} \rho\left(\mathbf{r}^{\prime}, t\right)+\frac{1}{c^{2}} \frac{\partial \mathbf{j}\left(\mathbf{r}^{\prime}, t\right)}{\partial t}\right) d^{3} \mathbf{r}^{\prime},
$$

where $\nabla^{\prime}=\partial / \partial \mathbf{r}^{\prime}$ and $G\left(\mathbf{r}-\mathbf{r}^{\prime}\right)=\exp \left(i \omega\left|\mathbf{r}-\mathbf{r}^{\prime}\right| / c\right) /\left|\mathbf{r}-\mathbf{r}^{\prime}\right|$ is the Green function. Further,

$$
\mathbf{j}(\mathbf{r}, t)=\int e \mathbf{v} \delta f(\mathbf{p}, \mathbf{r}, t) d^{3} \mathbf{p}, \quad \rho(\mathbf{r}, t)=\int e \delta f(\mathbf{p}, \mathbf{r}, t) d^{3} \mathbf{p} .
$$

Equations (2), (3), and (4) form a self-consistent set of equations (like Vlasov's equations for plasma [8]).

The first term in brackets in Eq. (3) corresponds to the contribution of the induced charges in metal, while the second term is for the induced currents. Using the charge conservation law $\partial \rho / \partial t+\nabla \mathbf{j}=0$, substituting Eq. (3) into Eq. (2), and integrating over the electron momentum $\int d^{3} \mathbf{p} e \mathbf{v}$, one obtains an equation for the current density

$$
\frac{\partial \mathbf{j}}{\partial t}+\gamma \mathbf{j}-e^{2}\left[\int_{\text {metal }} G\left(\mathbf{r}-\mathbf{r}^{\prime}\right)\left(-\int \nabla^{\prime}\left(\nabla^{\prime} \mathbf{j}\left(\mathbf{r}^{\prime}, t\right)\right) d t+\frac{1}{c^{2}} \frac{\partial \mathbf{j}\left(\mathbf{r}^{\prime}, t\right)}{\partial t}\right) d^{3} \mathbf{r}^{\prime}\right] \hat{\beta}=-e^{2} \mathbf{E}_{\mathrm{inc}} \hat{\beta},
$$

where $(\hat{\beta})_{j k}=\int\left(\partial f_{0} / \partial p_{j}\right) v_{k} d^{3} \mathbf{p}=-(n / m) \delta_{j k}$ and $n$ is the volume density of electrons. The resulting integrodifferential equation for $\mathbf{j}(\mathbf{r}, t)$ is

$$
\frac{\partial \mathbf{j}}{\partial t}+\gamma \mathbf{j}+\frac{\omega_{p}^{2}}{4 \pi} \int_{\text {metal }} G\left(\mathbf{r}-\mathbf{r}^{\prime}\right)\left(-\int \nabla^{\prime}\left(\nabla^{\prime} \mathbf{j}\left(\mathbf{r}^{\prime}, t\right)\right) d t+\frac{1}{c^{2}} \frac{\partial \mathbf{j}\left(\mathbf{r}^{\prime}, t\right)}{\partial t}\right) d^{3} \mathbf{r}^{\prime}=\frac{\omega_{p}^{2}}{4 \pi} \mathbf{E}_{\mathrm{inc}},
$$

where $\omega_{p}=\sqrt{4 \pi e^{2} n / m}$ is the plasma frequency. Owing to the skin-effect, electric current flows only near the metal surface. So, let $\mathbf{t}(\mathbf{r})$ be a tangential unit vector at the metal surface, so that $\mathbf{j}(\mathbf{r}, t)=\mathbf{t}(\mathbf{r}) j(\mathbf{r}, t)$. Multiplying Eq. (6) by $\mathbf{t}(\mathbf{r})$ and integrating over a specific metallic inclusion (call it $V_{1}$ ), we get to the equation for the average current density $\langle j\rangle=\left(1 / V_{1}\right) \int_{V_{1}} j(\mathbf{r}, t) d^{3} \mathbf{r}$. For monochromatic time dependence $\mathbf{j} \sim \exp (-i \omega t)$, it reads

$$
\begin{array}{r}
\omega^{2}\left(\langle j\rangle+\frac{\omega_{p}^{2}}{4 \pi c^{2} V_{1}} \int_{V_{1}} d^{3} \mathbf{r} \int_{\text {metal }} G\left(\mathbf{r}-\mathbf{r}^{\prime}\right) \mathbf{t}(\mathbf{r}) \cdot \mathbf{j}\left(\mathbf{r}^{\prime}, t\right) d^{3} \mathbf{r}^{\prime}\right)+i \omega \gamma\langle j\rangle \\
+\frac{\omega_{p}^{2}}{4 \pi V_{1}} \int_{V_{1}} d^{3} \mathbf{r} \int_{\text {metal }} G\left(\mathbf{r}-\mathbf{r}^{\prime}\right)\left(\mathbf{t}(\mathbf{r}) \nabla^{\prime}\right)\left(\nabla^{\prime} \mathbf{j}\left(\mathbf{r}^{\prime}, t\right)\right) d^{3} \mathbf{r}^{\prime}=i \omega \frac{\omega_{p}^{2}}{4 \pi V_{1}} \int_{V_{1}} \mathbf{t}(\mathbf{r}) \mathbf{E}_{\mathrm{inc}} d^{3} \mathbf{r} .
\end{array}
$$

The third term in the left-hand side of Eq. (7), on the second line of that equation, appears to be negative for the current oscillations, thus giving rise to resonant behavior of the MM response. When the current is determined from Eq. (7), the polarization of the metallic inclusion equals $\mathbf{P}=i \mathbf{j} / \omega$. Then one can apply the ordinary homogenization procedures [1-6].

If there are several metallic inclusions in the MM unit cell and the cells are periodically arranged, we need to take into account the inputs from all of the induced sources. For example, if there are two metallic inclusions, the kinetic equation (2) is written separately for them, each equation containing the induced charges and currents of both inclusions. So, one arrives at a system of coupled equations for such a MM. The case of split-ring chiral MMs, where each unit cell consists of two ring segments, was considered in [7].

\section{Application To Simple InClusion Geometries}

To illustrate the proposed approach, we apply the obtained results to determine the resonance frequency of localized plasmon polaritons in a single metallic element. First, consider a metallic bar with dimensions $a \gg b \gg$ $h$ the current mainly flows along the bar, so $\mathbf{t}=\mathbf{e}_{x}$. The current moves within the skin depth $\delta_{s}$ and vanishes at the ends of metallic bar $x= \pm a / 2$, therefore, $j(x)=j_{0} \delta_{s} \sin (\pi(x+a / 2) / a)[\delta(y-b / 2)+\delta(y+b / 2)]$. Then the resonant frequency is simply $f_{\text {res }}=c /(2 a)$. 
Now consider a ring segment of the length $R \alpha$ (see Fig. 1 (a), with the radius $R$ much greater than the width $d$ and thickness $h$ ). Similarly, one writes $\mathbf{j}=\mathbf{e}_{\varphi} j_{0} \delta_{s} \sin (\pi \varphi / \alpha)[\delta(r-R+d / 2)+\delta(r-R-d / 2)]$. The average polarization vector is of the form $\langle\mathbf{P}\rangle=i\langle\mathbf{j}\rangle / \omega$. The coefficient $j_{0}$ follows from the kinetic equation (7) as

$$
j_{0}=\frac{\left(i \omega \alpha / 2 h I_{1} \delta_{s}\right)\left(\int_{0}^{\alpha} \mathbf{e}_{\varphi} d \varphi\right) \mathbf{E}_{\text {inc }}}{\omega^{2} / c^{2}-\left(\pi^{2} / \alpha^{2} R^{2}\right)\left(1+\alpha I_{2} / \pi I_{1}\right)+i \omega \gamma 8 \alpha / \omega_{p}^{2} h d I_{1}},
$$

where $I_{1}>0$ and $I_{2}<0$ are integrals over angles $\varphi$ and radius $r$ :

$$
I_{1,2} \approx \frac{1}{d} \int_{R-d / 2}^{R+d / 2} d r \int_{0}^{\alpha} d \varphi^{\prime} \int_{0}^{\alpha} d \varphi \frac{r \sin \left(\Phi_{1,2}\right) \cos \left(\Phi_{2,1}\right)}{s\left(r, \varphi, \varphi^{\prime}\right)}, \quad \Phi_{1}=\frac{\pi \varphi^{\prime}}{\alpha}, \Phi_{2}=\varphi^{\prime}-\varphi,
$$

and $s\left(r, \varphi, \varphi^{\prime}\right)=\sqrt{r^{2}+(R-d / 2)^{2}-2(R-d / 2) r \cos \left(\varphi-\varphi^{\prime}\right)}$. The resonant frequency is then equal to $f_{\text {res }}=$ $(c / 2 \alpha R) \sqrt{1+\alpha I_{2} / \pi I_{1}}$. As seen in Fig. $1(\mathrm{~b})$, it takes lower values than the equivalent bar resonant frequency $c / 2 \alpha R$. A good agreement between the proposed model and full-wave numerical simulations is also seen.

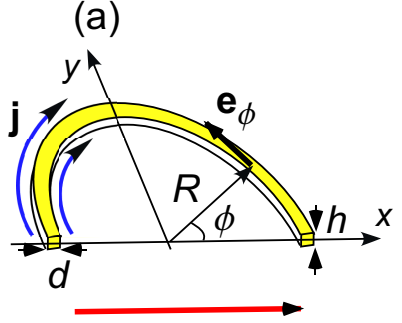

E

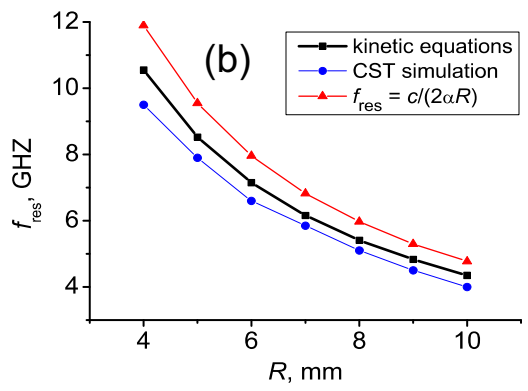

Fig. 1: (a) Schematic of a half-ring ( $\alpha=\pi)$ metallic inclusion showing its dimensions and the direction of the incident field. (b) Plot of $f_{\text {res }}(R)$ obtained from the proposed model vs. from full-wave numerical simulations (using CST Microwave Studio) for $\omega_{p}=1.38 \times 10^{16} \mathrm{rad} / \mathrm{s}, \gamma=40.7 \times 10^{12} \mathrm{~s}^{-1}, d=0.8 \mathrm{~mm}$, and $h=0.1 \mathrm{~mm}$. The resonance frequency for the equivalent bar, $f_{\text {res }}=c /(2 \alpha R)$, is also plotted.

\section{CONCLUSION}

We have proposed a technique for theoretical characterization of the electromagnetic response of MMs, based on the kinetic Boltzmann equation for the electrons in metal. The approach correctly reproduces the expected resonance behavior of single metallic inclusions. An explicit solution of the kinetic equation (6) can be obtained for more complicated shapes if the appropriate ansatz of the current density is assumed. This technique can be used to develop a unified $a b$ initio description of metal-dielectric MMs.

\section{REFERENCES}

[1] D. R. Smith, D. C. Vier, Th. Koschny, and C. M. Soukoulis, "Electromagnetic parameter retrieval from inhomogeneous metamaterials," Physical Review E, vol. 71, 036617, 2005.

[2] J. Petschulat, C. Menzel, A. Chipouline, C. Rockstuhl, A. Tunnermann, F. Lederer, and T. Pertsch, "Multipole approach to metamaterials," Physical Review A, vol. 78, 043811, 2008.

[3] A. Andryieuski, R. Malureanu, A. Lavrinenko, "Wave propagation retrieval method for metamaterials: Unambiguous restoration of effective parameters," Physical Review B, vol. 80, 193101, 2009.

[4] C. R. Simovski and S. A. Tretyakov, "On effective electromagnetic parameters of artificial nanostructured magnetic materials," Photonics and Nanostructures, vol. 8, p. 254-263, 2010.

[5] C. R. Simovski, "On electromagnetic characterization and homogenization of nanostructured metamaterials," Journal of Optics, vol. 13, 013001, 2011.

[6] A. Alù, "First-principles homogenization theory for periodic metamaterials," Physical Review B, vol. 84, 075153, 2011.

[7] A. V. Novitsky, V. M. Galynsky, and S. V. Zhukovsky, "Asymmetric transmission in planar chiral split-ring metamaterials: Microscopic Lorentz-theory approach," Physical Review B, vol. 86, 075138, 2012.

[8] E. M. Lifshits and L. P. Pitaevskii, Physical Kinetics, 2nd edition, Oxford, England: Pergamon Press, 1989.

[9] S. Raza, G. Toscano, A.-P. Jauho, M. Wubs, and N. A. Mortensen, "Unusual resonances in nanoplasmonic structures due to nonlocal response," Physical Review B, vol. 84, 121412(R), 2011. 